\title{
Variação nos níveis de prolina, diamina e poliaminas em cultivares de trigo submetidas a déficits hídricos ${ }^{(1)}$
}

\author{
Terezinha de Fátima Fumis ${ }^{(2)}$ e José Figueiredo Pedras ${ }^{(3)}$
}

\begin{abstract}
Resumo - O objetivo deste trabalho foi avaliar duas cultivares de trigo (Triticum aestivum L.) Anahuac e IAC-24, cultivadas em solo arenoso, sob três regimes de umidade do solo (-0,027 a -0,1 MPa; -0,027 a -0,045 MPa; e -0,027 MPa - irrigação constante). Foram analisadas as variações nos níveis de prolina, da diamina (putrescina) e das poliaminas (espermina e espermidina) e o teor relativo de água (TRA) durante o perfilhamento, emborrachamento, floração e no enchimento dos grãos. Nas plantas submetidas a déficits hídricos foi verificada menor TRA e acúmulo de prolina e putrescina; em relação às poliaminas não foram observadas diferenças entre os tratamentos. Nos tratamentos sob déficit hídrico, a cultivar IAC-24 apresentou teores mais elevados de prolina e putrescina, o mesmo sendo verificado com a cultivar Anahuac quando submetida a tratamento sem déficit hídrico.
\end{abstract}

Termos para indexação: Triticum aestivum, putrescina, espermidina, espermina, estresse hídrico.

Proline, diamine and polyamines accumulation in wheat cultivars submitted to water deficits

\begin{abstract}
The objective of this work was to evaluate two wheat (Triticum aestivum L.) cultivars, Anahuac and IAC-24, developed in sandy texture soil, under three levels of soil humidity ( -0.027 to $-0.1 \mathrm{MPa} ;-0.027$ to $-0.045 \mathrm{MPa}$; and $-0.027 \mathrm{MPa}$ - constantly irrigated). The variation in the levels of proline, diamine (putrescine) and polyamines (spermine and spermidine) content and the relative water content (RWC) during the tillering, booting, flowering and grain-filling was determined. The plants under water deficits presented smaller RWC and higher levels of proline and putrescine. In relation to the polyamines it wasn't observed difference among the treatments. The cv. IAC-24 under water deficit presented a higher content of proline and putrescine while the Anahuac showed the same results in the treatments without water deficit.
\end{abstract}

Index terms: Triticum aestivum, putrescine, spermidine, spermine, drought stress.

\section{Introdução}

As plantas, quando expostas a diversos tipos de estresse ambiental, notadamente o hídrico, podem apresentar acúmulo de prolina, putrescina e poliaminas. $\mathrm{O}$ acúmulo desses compostos sob déficit hídrico tem sido associado com a tolerância das plantas a essa condição desfavorável, podendo representar um mecanismo regulador da perda de água, mediante aumento da osmolaridade celular (potencial hídrico).

\footnotetext{
(1) Aceito para publicação em 18 de julho de 2001.

Extraído da tese de doutorado apresentada pelo primeiro autor à Universidade Estadual Paulista (Unesp), Botucatu, SP Parcialmente financiado pela Fundação da Unesp (Fundunesp).

(2) Unesp, Dep. de Ciências Biológicas, Caixa Postal 473 CEP 17033-360 Bauru, SP. E-mail: tffumis@fc.unesp.br

(3) Unesp, Caixa Postal 502, CEP 18618-000 Botucatu, SP E-mail: pedras@1aser.com.br
}

Sob a influência do estresse, a síntese de proteínas é inibida e a degradação de proteínas é acelerada, o que leva a um acúmulo de aminoácidos e aminas livres. Uma característica marcante de um distúrbio no metabolismo das proteínas é a mudança nas proporções dos aminoácidos e, freqüentemente, um aumento elevado na concentração de prolina (Larcher, 2000).

Vários autores relataram acúmulo de prolina quando as plantas foram submetidas ao déficit hídrico (Shevyakova, 1984; Hare \& Cress, 1997; Carceller et al., 1999; Lazcano-Ferrat \& Lovatt, 1999).

O acúmulo de prolina nas plantas sob estresse poderia ser decorrente da regulação osmótica e proteção da integridade celular (Stewart \& Lee, 1974; Shevyakova, 1984). Tal acúmulo teria também a função de proteger as células dos processos de desnaturação sob estresse hídrico e salino (Shevyakova, 1984), ou ainda participar na consti- 
tuição de um estoque de $\mathrm{N}$ e C que poderia ser utilizado depois do período de estresse (Taylor, 1996).

Embora vários trabalhos tenham relacionado a função protetora da prolina na adaptação das células sob estresse osmótico, ainda existem dúvidas se o acúmulo desse composto nos tecidos das plantas proporciona vantagem adaptativa ou simplesmente é uma conseqüência acidental de outros estresses, induzindo mudanças no metabolismo (Hare \& Cress, 1997).

A diamina (putrescina) e as poliaminas (espermidina e espermina) estão presentes em inúmeras etapas do crescimento e diferenciação das plantas, como a germinação, divisão celular, diferenciação das folhas, flores e raízes, desenvolvimento da flor e fruto; senescência dos órgãos e outros (Galston \& KaurSawhney, 1995). Em vários tipos de estresse ambiental, principalmente o hídrico, verificou-se acúmulo desses compostos (Flores \& Galston, 1982b), principalmente em relação a putrescina (Aziz \& Larher, 1995; Zhang et al., 1996; Aziz et al.,1997), todavia ainda não está claro em que circunstâncias esses compostos contribuem para a adaptação das plantas ao estresse (Aziz \& Larher, 1995; Galston et al., 1997).

$\mathrm{O}$ aumento tanto nos níveis de putrescina e prolina poderiam estar associados nas plantas, podendo o aumento deles estar relacionados com a resistência à seca (Aziz \& Larher, 1995).

O objetivo deste trabalho foi avaliar as variações de prolina, diamina (putrescina) e poliaminas (espermidina e espermina) em cultivares de trigo submetidas a diferentes regimes de umidade.

\section{Material e Métodos}

O trabalho foi realizado em casa de vegetação no Departamento de Ciências Biológicas da Faculdade de Ciências do Campus de Bauru, SP, Unesp, no período de maio a setembro de 1995 . Utilizaram-se as cultivares de trigo (Triticum aestivum L.) Anahuac e IAC-24.

Foram utilizados 36 vasos por cultivar. Cada vaso, de plástico impermeável, recebeu $10 \mathrm{~kg}$ de terra de textura arenosa. Para minimizar os efeitos da evaporação e melhorar a uniformidade entre os tratamentos, os vasos foram cobertos com plástico flexível transparente. No controle das irrigações, os vasos eram pesados diariamente, para posterior reposição da água evapotranspirada no período, sempre que necessário.
A partir do 18 o dia após o transplantio, teve início a diferenciação dos tratamentos, em relação aos três regimes hídricos: -0,027 a -0,1 MPa; $-0,027$ a $-0,045 \mathrm{MPa}$; e $-0,027 \mathrm{MPa}$ (constantemente irrigado).

$\mathrm{Na}$ determinação de prolina, usou-se o método de Bates et al. (1973) modificado por Torello \& Rice (1986), e na determinação da diamina e poliaminas, o método de Flores \& Galston (1982a), cujos valores foram quantificados por espectroscopia de fluorescência. O teor relativo de água (TRA) foi avaliado segundo Barrs (1968), a partir da retirada dos discos da folha. As determinações de prolina, diamina, poliaminas e TRA foram realizadas nas folhas de trigo mais novas, completamente desenvolvidas.

O delineamento experimental foi inteiramente casualizado, em esquema fatorial $2 \times 3 \times 4$, correspondente a duas cultivares de trigo (Anahuac e IAC-24), três regimes de umidade do solo e quatro épocas de coleta de plantas $(30,50,70$ e 90 dias após o transplantio, correspondendo, respectivamente, ao perfilhamento, emborrachamento, florescimento e formação de grãos), com três repetições e duas plantas por coleta.

Os resultados obtidos foram submetidos à análise de variância e as médias comparadas pelo teste de Tukey, a $5 \%$ de probabilidade.

\section{Resultados e Discussão}

As variáveis prolina, putrescina, poliaminas e o teor relativo de água apresentaram significância estatística entre cultivares, tratamentos e coletas, e na interação entre cultivares e tratamentos e coletas.

Em relação aos níveis de prolina, houve efeito significativo em todos os tratamentos nos quatro estádios de desenvolvimento das duas cultivares (Tabela 1). Os menores valores foram observados no tratamento constantemente irrigado e aumentou à medida que diminuiu a umidade do solo. No tratamento mais úmido, a cv. Anahuac apresentou teores mais elevados de prolina; enquanto nos demais tratamentos, com exceção do tratamento T1(-0,027 a -0,1 MPa), aos 90 dias após transplantio, apresentou níveis menores, quando comparado com a cv. IAC-24.

O aumento do déficit hídrico promoveu incremento nos teores de prolina nas folhas das plantas (Tabela 1). Resultados semelhantes foram obtidos para o algodão (McMichael \& Elmore, 1977); trigo (Heerden \& Villiers, 1996; Strauss \& Agenbag, 1998); feijão (Lazcano-Ferrat \& Lovatt, 1999); e milho (Carceller et al., 1999).

O aumento dos teores de prolina nas plantas de trigo sob déficit hídrico poderia ser atribuído ao 
aumento das atividades das enzimas envolvidas na síntese deste aminoácido (Kandpal \& Rao, 1982) ou à inibição da oxidação da prolina (Kiyosue et al., 1996).

A cultivar Anahuac, no tratamento mais úmido, apresentou menores variações nos teores de prolina,

Tabela 1. Comparação das médias de tratamentos, em cada coleta, de duas cultivares de trigo (Anahuac e IAC-24) submetidas a três regimes hídricos: T1 $(-0,027 \mathrm{a}-0,1 \mathrm{MPa})$, T2 (-0,027 a -0,045 MPa) e T3 (-0,027 MPa-irrigado $)^{(1)}$.

\begin{tabular}{|c|c|c|c|c|c|}
\hline \multirow[t]{2}{*}{ Cultivar } & \multirow[t]{2}{*}{ Tratamento } & \multicolumn{4}{|c|}{ Coleta (dias após transplantio) } \\
\hline & & 30 & 50 & 70 & 90 \\
\hline & & \multicolumn{4}{|c|}{ Prolina $\left(\mu \mathrm{mol} / \mathrm{g} \mathrm{ms} 10^{-3}\right)$} \\
\hline \multirow[t]{3}{*}{ Anahuac } & $\mathrm{T} 1$ & $26,79 \mathrm{Ab}$ & $31,41 \mathrm{Ab}$ & $63,78 \mathrm{Ab}$ & $94,54 \mathrm{Aa}$ \\
\hline & $\mathrm{T} 2$ & $18,77 \mathrm{Bb}$ & $24,71 \mathrm{Bb}$ & $38,62 \mathrm{Bb}$ & $58,13 \mathrm{Bb}$ \\
\hline & $\mathrm{T} 3$ & $11,22 \mathrm{Ca}$ & $14,66 \mathrm{Ca}$ & $19,45 \mathrm{Ca}$ & $24,64 \mathrm{Ca}$ \\
\hline Média & & 18,93 & 23,59 & 40,62 & 59,10 \\
\hline \multirow[t]{3}{*}{ IAC-24 } & $\mathrm{T} 1$ & $62,01 \mathrm{Aa}$ & $70,53 \mathrm{Aa}$ & $79,48 \mathrm{Aa}$ & $80,63 \mathrm{Ab}$ \\
\hline & $\mathrm{T} 2$ & $44,96 \mathrm{Ba}$ & $42,77 \mathrm{Ba}$ & $62,21 \mathrm{Ba}$ & $73,82 \mathrm{Ba}$ \\
\hline & $\mathrm{T} 3$ & $8,60 \mathrm{Ca}$ & $9,88 \mathrm{Cb}$ & $9,58 \mathrm{Cb}$ & $19,09 \mathrm{Cb}$ \\
\hline \multirow[t]{2}{*}{ Média } & & 38,52 & 41,06 & 50,42 & 57,85 \\
\hline & & \multicolumn{4}{|c|}{ Putrescina (nmol/g mf) } \\
\hline \multirow[t]{3}{*}{ Anahuac } & $\mathrm{T} 1$ & $2.683,06 \mathrm{Ab}$ & $3.699,45 \mathrm{Ab}$ & $4.661,18 \mathrm{Ab}$ & $5.890,71 \mathrm{Ab}$ \\
\hline & $\mathrm{T} 2$ & $1.333,33 \mathrm{Bb}$ & $1.595,63 \mathrm{Bb}$ & $3.240,44 \mathrm{Bb}$ & $4.371,55 \mathrm{Bb}$ \\
\hline & T3 & $853,90 \mathrm{Ca}$ & $1.202,18 \mathrm{Ba}$ & $1.857,92 \mathrm{Ca}$ & $2.278,69 \mathrm{Ca}$ \\
\hline Média & & $1.623,43$ & $2.165,75$ & $3.253,18$ & $4.180,32$ \\
\hline \multirow[t]{3}{*}{ IAC-24 } & $\mathrm{T} 1$ & $4.284,29 \mathrm{Aa}$ & $5.109,29 \mathrm{Aa}$ & $5.715,41 \mathrm{Aa}$ & $6.277,05 \mathrm{Aa}$ \\
\hline & $\mathrm{T} 2$ & $2.541,01 \mathrm{Ba}$ & $3.431,69 \mathrm{Ba}$ & $3.814,09 \mathrm{Ba}$ & $4.816,39 \mathrm{Ba}$ \\
\hline & T3 & $547,54 \mathrm{Ca}$ & $1.013,22 \mathrm{Ca}$ & $1.480,87 \mathrm{Cb}$ & $21.29,51 \mathrm{Ca}$ \\
\hline \multirow[t]{2}{*}{ Média } & & $2.457,61$ & $3.184,73$ & $3.670,12$ & $4.407,65$ \\
\hline & & \multicolumn{4}{|c|}{ Espermidina (nmol/g mf) } \\
\hline \multirow[t]{3}{*}{ Anahuac } & $\mathrm{T} 1$ & $79,43 \mathrm{Cb}$ & $109,80 \mathrm{Aa}$ & $124,82 \mathrm{Aa}$ & $205,93 \mathrm{Aa}$ \\
\hline & $\mathrm{T} 2$ & $165,95 \mathrm{Ba}$ & $94,56 \mathrm{Bb}$ & $122,24 \mathrm{Aa}$ & $119,20 \mathrm{Ba}$ \\
\hline & T3 & $219,35 \mathrm{Aa}$ & $139,37 \mathrm{Aa}$ & $115,47 \mathrm{Aa}$ & $88,56 \mathrm{Ca}$ \\
\hline Média & & 154,81 & 114,58 & 120,84 & 137,90 \\
\hline \multirow[t]{3}{*}{ IAC-24 } & $\mathrm{T} 1$ & $108,75 \mathrm{Ca}$ & $88,62 \mathrm{Ca}$ & $80,06 \mathrm{Ab}$ & $49,59 \mathrm{Bb}$ \\
\hline & $\mathrm{T} 2$ & $143,84 \mathrm{Ba}$ & $148,09 \mathrm{Ba}$ & $80,85 \mathrm{Ab}$ & $37,10 \mathrm{Bb}$ \\
\hline & T3 & $200,25 \mathrm{Aa}$ & $130,60 \mathrm{Aa}$ & $90,78 \mathrm{Aa}$ & $83,68 \mathrm{Aa}$ \\
\hline \multirow[t]{2}{*}{ Média } & & 150,95 & 122,44 & 83,90 & 56,79 \\
\hline & & \multicolumn{4}{|c|}{ Espermina (nmol/g mf) } \\
\hline \multirow[t]{3}{*}{ Anahuac } & $\mathrm{T} 1$ & $83,63 \mathrm{Aa}$ & $97,18 \mathrm{Ba}$ & $104,01 \mathrm{Ba}$ & $247,81 \mathrm{Aa}$ \\
\hline & $\mathrm{T} 2$ & $114,68 \mathrm{Ab}$ & $112,73 \mathrm{Aa}$ & $145,77 \mathrm{Aa}$ & $217,69 \mathrm{Aa}$ \\
\hline & $\mathrm{T} 3$ & $99,12 \mathrm{Aa}$ & $71,91 \mathrm{Ba}$ & $99,12 \mathrm{Ba}$ & $136,05 \mathrm{Ba}$ \\
\hline Média & & 99,14 & 93,94 & 116,30 & 200,52 \\
\hline \multirow[t]{3}{*}{ IAC-24 } & $\mathrm{T} 1$ & $64,14 \mathrm{Ca}$ & $81,63 \mathrm{Ba}$ & $97,18 \mathrm{Ba}$ & $124,39 \mathrm{Ab}$ \\
\hline & $\mathrm{T} 2$ & $188,53 \mathrm{Ba}$ & $114,68 \mathrm{Aa}$ & $163,36 \mathrm{Aa}$ & $143,83 \mathrm{Ab}$ \\
\hline & T3 & $99,12 \mathrm{Aa}$ & $91,57 \mathrm{Ba}$ & $101,07 \mathrm{Ba}$ & $42,76 \mathrm{Bb}$ \\
\hline \multirow[t]{2}{*}{ Média } & & 117,26 & 95,96 & 120,54 & 103,66 \\
\hline & & \multicolumn{4}{|c|}{ Teor relativo de água $(\%)$} \\
\hline \multirow[t]{3}{*}{ Anahuac } & $\mathrm{T} 1$ & $71,06 \mathrm{Ba}$ & $68,44 \mathrm{Ca}$ & $64,82 \mathrm{Bb}$ & $60,36 \mathrm{Bb}$ \\
\hline & $\mathrm{T} 2$ & $73,99 \mathrm{Bb}$ & $74,32 \mathrm{Bb}$ & $66,32 \mathrm{Bb}$ & $64,81 \mathrm{Bb}$ \\
\hline & $\mathrm{T} 3$ & $82,88 \mathrm{Aa}$ & $79,03 \mathrm{Ab}$ & $78,96 \mathrm{Ab}$ & 93,40Aa \\
\hline Média & & 75,98 & 73,93 & 70,03 & 72,86 \\
\hline \multirow[t]{3}{*}{ IAC-24 } & $\mathrm{T} 1$ & $69,13 \mathrm{Ba}$ & $71,31 \mathrm{Ca}$ & $70,13 \mathrm{Ca}$ & $78,43 \mathrm{Ba}$ \\
\hline & $\mathrm{T} 2$ & $80,67 \mathrm{Aa}$ & $81,38 \mathrm{Ba}$ & $78,28 \mathrm{Ba}$ & $81,78 \mathrm{Ba}$ \\
\hline & T3 & $84,69 \mathrm{Aa}$ & $89,03 \mathrm{Aa}$ & $82,89 \mathrm{Aa}$ & $95,15 \mathrm{Aa}$ \\
\hline Média & & 78,16 & 80,57 & 77,10 & 85,12 \\
\hline
\end{tabular}

(1)Médias seguidas da mesma letra, maiúscula na coluna, dentro de cada cultivar, e minúscula na coluna, dentro do mesmo tratamento, não diferem entre si pelo teste de Tukey a 5\% de probabilidade; ms: matéria seca; $\mathrm{mf}$ : matéria fresca. mas ainda assim um pouco mais elevados que os observados em relação a IAC-24. No tratamento submetido ao maior déficit hídrico, verificaram-se, no início, valores menores de prolina com aumento gradativo no decorrer do ciclo. Apenas na fase de produção de grãos houve maior acúmulo desse composto. A cv. IAC-24 quando submetida ao mesmo tratamento apresentou valores elevados de prolina no início, permanecendo constante com o decorrer das coletas (Tabela 1). Portanto, sob déficit hídrico, a cv. IAC-24 apresentou melhor aclimatação ao estresse.

O acúmulo de prolina durante o estresse hídrico pode ser um indicador potencial de tolerância à seca em cultivares de trigo (Heerden \& Villiers, 1996).

Foi observado entre a putrescina e a espermidina e espermina resultados significativos dos três regimes hídricos, principalmente em relação a putrescina (Tabela 1).

Nas duas cultivares, os níveis de putrescina aumentaram quando as plantas foram submetidas a déficits hídricos; a cv. IAC-24 apresentou os maiores níveis em todas as coletas em relação aos tratamentos sob estresse, e somente no tratamento irrigado a cv. Anahuac teve teores um pouco mais elevados da amina, embora não significativos, à exceção do verificado aos 70 dias após o transplantio (Tabela 1). Os níveis de espermidina e espermina foram menores que o de putrescina e não apresentaram diferenças tão nítidas em relação aos tratamentos e coletas quando comparados com a putrescina (Tabela 1). Os resultados relativos à putrescina estão de acordo com os verificados com aveia, cevada, milho e trigo (Flores \& Galston, 1982b, 1984); canade-açúcar (Zhang et al., 1996); e Brassica napus L. (Aziz et al., 1997) quando submetidos a déficits hídricos.

Flores \& Galston (1982b) relataram aumento de putrescina nas plantas de aveia expostas ao estresse osmótico, com um gradual declínio no conteúdo de espermina e espermidina. Resultados semelhantes foram verificados por esses autores em relação ao trigo, o mesmo ocorrendo com Xu et al. (1995); Zhang et al. (1996), com cana-de-açúcar; e Aziz et al. (1997), com Brassica napus L. No presente experimento o mesmo comportamento foi observado em relação aos níveis de espermidina, principalmente na cv. IAC-24. 
Nas plantas sob déficit hídrico, os valores do TRA obtidos ao longo do ciclo foram menores, notadamente na cv. Anahuac, que apresentou efeitos significativos entre os tratamentos (Tabela 1). A cv. IAC-24 apresentou teores pouco variáveis no decorrer do experimento enquanto na Anahuac houve declínio (Tabela 1). Esta resposta da IAC-24 poderia indicar sua adaptação ao déficit hídrico através de sua maior capacidade de ajustamento osmótico, como verificado em plantas de trigo por Denadai (1989).

A cv. Anahuac apresentou correlações significativas negativas quanto a TRA e prolina nos dois tratamentos submetidos ao estresse hídrico: -0,027 a $-0,1 \mathrm{MPa}\left(\mathrm{r}=-0,923^{*}\right)$ e $-0,027$ a $-0,045 \mathrm{MPa}$ $\left(r=-0,836^{*}\right)$, resultado semelhante foi obtido em plantas de feijão submetidas a déficit hídrico (LazcanoFerrat \& Lovatt, 1999). Por outro lado, a cv. IAC-24 não apresentou correlações significativas, nos três tratamentos, entre as duas variáveis analisadas. Assim como verificado quanto à prolina, apenas a cv. Anahuac apresentou correlações significativas negativas de TRA e putrescina, e também somente nos tratamentos submetidos a déficit hídrico: $-0,027$ a $-0,1 \mathrm{MPa}\left(\mathrm{r}=-0,970^{*}\right)$ e $-0,027$ a $-0,045 \mathrm{MPa}$ $\left(r=-0,891^{*}\right)$.

Quando comparados os dados de prolina e putrescina com o TRA, verificou-se que o aumento desses compostos teria facilitado o ajustamento osmótico, sendo a IAC-24 a que apresentou melhor adaptação às condições de estresse, pelos valores constantes de prolina e putrescina e de TRA encontrados. A ocorrência do ajustamento osmótico já no início do déficit hídrico poderia ter favorecido o melhor desempenho desta cultivar. Resultado que pode ser confirmado com a redução de $63,4 \%$ e $83,5 \%$ na produção de grãos, da cv. Anahuac, nos tratamentos submetidos ao menor déficit hídrico e ao mais elevado; e de $39,1 \%$ e $74,1 \%$ da cv. IAC-24, respectivamente, em relação ao tratamento irrigado constantemente.

\section{Conclusões}

1. A cv. IAC-24 é mais adaptada às condições de estresse hídrico.
2. O aumento de prolina e putrescina nas plantas de trigo é indicador de estresse hídrico, na cv. Anahuac, e protetor de estresse hídrico, na cv. IAC-24.

\section{Agradecimentos}

À Fundunesp, pelo suporte financeiro; à Dra. Jurema Schons e Dra. Sheila Zambello de Pinho, pela contribuição na realização deste trabalho.

\section{Referências}

AZIZ, A.; LARHER, F. Changes in polyamine titers associated with the proline response and osmotic adjustment of rape leaf disc submitted to osmotic stresses. Plant Science, Limerick, v. 112, p. 175-186, 1995.

AZIZ, A.; MARTIN-TANGUY, J.; LARHER, F. Plasticity of polyamine metabolism associated with high osmotic stress in rape leaf discs and with ethylene treatment. Plant Growth Regulation, Dordrecht, v. 21, p. 153-163, 1997.

BARRS, H. D. Effect of cycle variations in gas exchange under constant environmental conditions on the ratio of transpiration to net photosynthesis. Physiologia Plantarum, Copenhagen, v. 21, p. 918-929, 1968.

BATES, L. S.; WALDREN, R. P.; TEARE, I. D. Rapid determination of free proline for water-stress studies. Plant and Soil, Dordrecht, v. 39, p. 205-207, 1973.

CARCELLER, M.; PRYSTUPA, P.; LEMCOFF, J. H. Remobilization of proline and other nitrogen compounds from senescing leaves of maize under water stress. Journal of Agronomy and Crop Science, Berlin, v. 183, p. 6166, 1999.

DENADAI, I. A. M. Avaliação de alguns parâmetros fisiológicos relacionados à resistência à seca em quatro cultivares de trigo. 1989. 93 f. Dissertação (Mestrado) - Universidade Estadual Paulista, Botucatu. 1989.

FLORES, H. E.; GALSTON, A. W. Analysis of polyamines in higher plants by high performance liquid chromatography. Plant Physiology, Bethesda, v. 9, p. 701706,1982 a.

FLORES, H. E.; GALSTON, A. W. Polyamines and plant stress: activation of putrescine biosynthesis by osmotic shock. Science, Washington, v. 217, p. 1259-1261, 1982b. 
FLORES, H. E.; GALSTON, A. W. Osmotic stressinduced polyamine accumulation in cereal leaves. II. Relation to amino acid pools. Plant Physiology, Bethesda, v. 75 , p. $110-113,1984$

GALSTON, A. W.; KAUR-SAWHNEY, R. Polyamines as endogenous growth regulators. In: DAVIES, P. J. (Ed.). Plant hormones physiology, biochemistry and molecular biology. Dordrecht: Kluwer Academic, 1995. p. 280-295.

GALSTON, A. W.; KAUR-SAWHNEY, R.; ALTABELLA, T.; TIBURCIO, A. F. Plant polyamines in reproductive activity and response to abiotic stress. Botanica Acta, Stuttgart, v. 110, p. 197-207, 1997.

HARE, P. D.; CRESS, W. A. Metabolic implications of stress induced proline accumulation in plants. Plant Growth Regulation, Dordrecht, v. 21, p. 79-102, 1997.

HEERDEN, P. D. R.; VILLIERS, O. T. Evaluation of proline accumulation as an indicator of drought tolerance in spring wheat cultivars. South African Journal of Plant and Soil, Pretoria, v. 13, p. 17-21, 1996.

KANDPAL, R. P.; RAO, N. A. Water stress induced alterations in the properties of ornithine aminotransferase from ragi (Eleusine coracana) leaves. Biochemistry International, Marvickville, v. 5, p. 297-302, 1982.

KIYOSUE,T.; YOSHIBA, Y.; YAMAGUCHISHINOZAKI, K.; SHINOZAKI, K. A nuclear gene encoding mitochondrial proline dehydrogenase: an enzyme involved in proline metabolism, is upregulated by proline but down regulated by dehydration in Arabidosis. Plant Cell, Rockville, v. 8, p. 1323-1335, 1996.

LARCHER, W. Ecofisiologia vegetal. São Carlos: RiMa, 2000. $531 \mathrm{p}$.

LAZCANO-FERRAT, I.; LOVATT, C. J. Relationship between relative water content, nitrogen pools, and growth of Phaseolus vulgaris L. and P. acutifolius A. Gray during water deficit. Crop Science, Madison, v. 39, p. 467-475, 1999.

McMiCHAEL, B. L.; ElMORE, C. D. Proline accumulation in water stressed cotton leaves. Crop Science, Madison, v. 17, p. 905-908, 1977.

SHEVYAKOVA, N. I. Metabolism and the physiological role of proline in plants under conditions of water and salt stress. Soviet Plant Physiology, New York, v. 30, p. 597 608, 1984.

STEWART, G. R.; LEE, J. A. The role of proline accumulation in halophytes. Planta, Berlin, v. 120, p. 279289,1974

STRAUSS, J. A.; AGENBAG, G. A. A comparison of two methods of inducing water stress in wheat (Triticum aestivum L.). South African Journal of Plant and Soil, Pretoria, v. 15, p. 121-122, 1998.

TAYLOR, C. B. Proline and water deficit: ups, downs, ins, and outs. Plant Cell, Rockville, v. 8, p. 1221-1224, 1996.

TORELLO, W. A.; RICE, L. A. Effects of $\mathrm{NaCl}$ stress on proline and cation accumulation in salt sensitive tolerance turfgrass. Plant and Soil, Dordrecht, v. 93, p. 241-247, 1986.

XU, Z. Z.; YU, Z. W.; QI, X. H.; YU, S. L. Effect of soil drought on ethylene evolution, polyamine accumulation and cell membranes in the leaf of winter wheat. Acta Phytophysiologica Sinica, Beijing, v. 21, p. 295-301, 1995.

ZHANG, M. Q.; CHENG, R. K.; YU, S. L. Changes of polyamine metabolism in drought-stressed sugarcane leaves and their relation to drought resistance. Acta Phytophysiologica Sinica, Beijing, v. 22, p. 377-382, 1996. 